\title{
Erratum to: Transitivity of generic semigroups of area-preserving surface diffeomorphisms
}

\author{
Andres Koropecki · Meysam Nassiri
}

Published online: 15 January 2011

(C) Springer-Verlag 2011

\section{Erratum to: Math. Z. (2010) 266:707-718 DOI 10.1007/s00209-009-0595-7}

Theorem 12 in [2] claims that for a Moser generic diffeomorphism $f$ and an invariant residual domain $U$, there are no periodic points in the boundary of $U$. This is claimed to be a direct consequence of [3]. However, Mather's theorem is weaker than that; specifically, it says that the rotation number of the prime ends compactification on any end of $U$ is irrational.

On the sphere, Mather's result implies what is claimed in Theorem 12, and the proof (which can be found in [1]) uses the existence of homoclinic intersections for periodic points of $f$ guaranteed by a theorem of Pixton. This can also be done in $\mathbb{T}^{2}$ using [4]. However, for higher genus, the claim remains open.

We will prove a weaker version of Theorem 12 of [2]. To do this, we define for each $f$ a $C^{r}$-residual set $\mathcal{G}_{f}$, and we prove that the statement of Theorem 12 of [2] holds if we additionally require that the residual domain $U$ be $g$-invariant for some $g \in \mathcal{G}_{f}$. This is enough for our proofs. The modifications needed in the statements and proofs of [2] are as follows:

1. In Lemmas 17 and 18, " $f$ and $g$ are Moser generic" should be changed to " $f$ is Moser generic and $g \in \mathcal{G}_{f}$ ", where $\mathcal{G}_{f}$ is the residual set from Definition 2 below. The proofs remain the same, using Proposition 5 from this article instead of Theorem 12 of [2];

Andres Koropecki was partially supported by FAPERJ-Brasil.

The online version of the original article can be found under doi:10.1007/s00209-009-0595-7.

\footnotetext{
A. Koropecki $(\varangle)$

Instituto de Matemática, Universidade Federal Fluminense, Rua Mário Santos Braga S/N, Niterói, RJ 24020-140, Brazil e-mail: koro@mat.uff.br

M. Nassiri

School of Mathematics, Institute for Research in Fundamental Sciences (IPM),

P. O. Box 19395-5746, Tehran, Iran

e-mail: nassiri@impa.br
} 
2. In the proof of Lemma 19, Proposition 4 of the present article should be used instead of Theorem 12 of [2] (because frontiers have planar neighborhoods);

3. The set $\mathcal{R}_{f}$ defined in the proof of Theorem 23 should be intersected with $\mathcal{G}_{f}$ (which still gives a residual set).

As in [2], we assume from now on that $S$ is a compact orientable surface and $r \geq 16$, so that Moser genericity is a $C^{r}$-generic condition. From [3, Theorem 5.2], if $f \in \operatorname{Diff}_{\omega}^{r}(S)$ is Moser generic and $p$ is a hyperbolic periodic point, then any two branches of $p$ have the same closure (a branch is a connected component of $W_{f}^{s}(p) \backslash p$ or $\left.W_{f}^{u}(p) \backslash p\right)$. Thus if we define $K_{p, f}=\overline{W_{f}^{s}(p)}$, we have that $K_{p, f}$ is the closure of any stable or unstable branch of $p$.

Proposition 1 For a Moser generic $f \in \operatorname{Diff}_{\omega}^{r}(S)$, if $p$ is a hyperbolic periodic point and $K_{p, f}$ has a planar neighborhood, then $p$ has a homoclinic intersection.

Proof The proof of [5] assumes that the whole manifold is planar, but it works almost without modification if instead we assume that the stable and unstable manifolds of $p$ are contained in a planar submanifold of $S$.

This is clear from the proof presented in [4] (the first part of the proof of Theorem 2, p. 582, dealing only with the sphere) since it is a purely topological argument taking place in a neighborhood of the union of the stable and unstable manifolds of $p$, and the only part where the dynamics is relevant is in a local argument in a neighborhood of $p$. In our setting, using the fact that $K_{p, f}$ is the closure of both the stable and unstable manifolds of $p$, we can restrict our attention to a planar neighborhood of $p$, and by embedding this neighborhood in a sphere the same proof applies.

Definition 2 For a Moser generic $f$, we denote by $\mathcal{G}_{f}$ the set of all Moser generic $g \in$ $\operatorname{Diff}_{\omega}^{r}(S)$ such that the following property holds: for any hyperbolic periodic point $p$ of $f$ and $k>0$,

$$
\text { if } K_{p, f} \cap g^{k}\left(K_{p, f}\right) \neq \varnothing \text { then } W_{f}^{s}(p) \pitchfork g^{k}\left(W_{f}^{s}(p)\right) \neq \emptyset \neq W_{f}^{u}(p) \pitchfork g^{k}\left(W_{f}^{s}(p)\right)
$$

where $A \pitchfork B$ stands for the set of transversal intersections of $A$ and $B$.

Proposition 3 If $f$ is Moser generic, then $\mathcal{G}_{f}$ is $C^{r}$-residual.

Proof Fix a Moser generic $g \in \operatorname{Diff}_{\omega}^{r}(S), k \in \mathbb{N}$, and a hyperbolic periodic point $p$ of $f$. Since the set $\operatorname{Per}_{k}(g)$ of periodic points of $g$ with period at most $k$ is finite, using a perturbation of $g$ of the form $h g h^{-1}$ with $h$ close to the identity we may assume that $\operatorname{Per}_{k}(g) \cap$ д $K_{p, f}=\varnothing$ (because $\partial K_{p, f}$ has empty interior). Suppose $K_{p, f}$ intersects $g^{k}\left(K_{p, f}\right)$. Since $g$ preserves area and $K_{p, f}$ is connected, this implies that $g^{k}\left(K_{p, f}\right) \cap \partial K_{p, f} \neq \emptyset$. Choose $x \in g^{k}\left(K_{p, f}\right) \cap \partial K_{p, f}$, and note that $x \notin \operatorname{Per}_{k}(g)$. Let $x_{n} \in W_{f}^{s}(p)$ and $y_{n} \in W_{f}^{s}(p)$ be such that $x_{n} \rightarrow x$ and $g^{k}\left(y_{n}\right) \rightarrow x$. Let $U$ be a neighborhood of $x$ such that $g^{i}(U)$ is disjoint from $U$ for $-k \leq i \leq 0$. By standard arguments we may choose, for any sufficiently large $n$, a map $\tilde{h} \in \operatorname{Diff}_{\omega}^{r}(S)$ supported in $g^{-1}(U)$ and $C^{r}$-close to the identity such that $\tilde{h}\left(g^{k-1}\left(y_{n}\right)\right)=g^{-1}\left(x_{n}\right)$. Letting $\tilde{g}=g \tilde{h}$ we obtain a map $C^{r}$-close to $g$ such that $\tilde{g}^{k}\left(y_{n}\right)=$ $x_{n} \in W_{f}^{s}(p)$. Thus $\tilde{g}^{k}\left(W_{f}^{s}(p)\right) \cap W_{f}^{s}(p) \neq \emptyset$, and this intersection can be made transverse with a perturbation of $\tilde{g}$. Note that once the intersection is transverse, it persists under new perturbations. Since $K_{p, f}$ is the closure of $W_{f}^{u}(p)$ as well, and $\tilde{g}^{k}\left(K_{p, f}\right) \cap K_{p, f} \neq \emptyset$, we may use the same argument to obtain a perturbation $\hat{g}$ of $\tilde{g}$ which also has a point of transversal intersection between $W_{f}^{u}(p)$ and $\hat{g}^{k}\left(W_{f}^{s}(p)\right)$. This shows that, for $p$ and $k$ fixed, the 
set of maps $g$ satisfying (1) is dense in $\operatorname{Diff}_{\omega}^{r}(S)$. Since condition (1) is also open, the set $\mathcal{U}_{p, k} \subset \operatorname{Diff}_{\omega}^{r}(S)$ where property (1) holds for this choice of $p$ and $k$ is open and dense. Since $\mathcal{G}_{f}$ is the intersection of the sets $\mathcal{U}_{p, k}$ over all $k \in \mathbb{N}$ and all hyperbolic periodic points $p$ of $f$ (which are countably many), this completes the proof.

Recall that a residual domain is a connected component of the complement of a nontrivial continuum. By [3, Lemma 2.3], a residual domain has finitely many boundary components.

Proposition 4 Let $f$ be Moser generic, $U$ a periodic connected open set and $K \subset \partial U a$ periodic nontrivial continuum. If $K$ has a planar neighborhood, then $K$ contains no periodic points.

Proof Let $n$ be such that $f^{n}(K)=K$ and $f^{n}(U)=U$. Suppose that there is a periodic point $p$ of $f$ in $K$. We assume that $f^{n}(p)=p$, by increasing $n$ if necessary. Since $f$ (and so $f^{n}$ ) is Moser generic, $p$ is either elliptic (Moser stable) or hyperbolic. By an argument of Mather, we can show that $p$ is hyperbolic: suppose on the contrary that $p$ is Moser stable. Then there is a sequence of invariant disks converging to $p$ such that the dynamics of $f^{n}$ on the boundary of each disk is minimal. If $D$ is such a disk and $K$ intersects $\partial D$, then ว $D \subset K \subset \partial U$, which implies by connectedness that $U$ is either contained in $D$ or disjoint form $D$. The first case is not possible if we choose $D$ small enough, while the second case contradicts the fact that $p \in \partial U$. Thus $p$ is hyperbolic.

By [3, Proposition 11.1] it follows that $W_{f}^{s}(p)$ and $W_{f}^{u}(p)$ are contained in $K$. Since $K$ has a planar neighborhood, it follows from Proposition 1 that $p$ has a homoclinic intersection. By the $\lambda$-lemma, this implies that there is a decreasing sequence of rectangles $R_{i}$ with boundary in $W_{f}^{s}(p) \cup W_{f}^{u}(p) \subset K$ accumulating on $p$. This contradicts the connectedness of $U$ : in fact $U$ cannot be contained in all of the rectangles $R_{i}$, but by connectedness if $U$ is not contained in $R_{i}$ then $\bar{U}$ is disjoint from the interior of $R_{i+1}$, which contains points of $\partial U$. This is a contradiction.

Proposition 5 Let $f$ be Moser generic, and let $U$ be an $f$-periodic residual domain. If $U$ is also g-periodic for $g \in \mathcal{G}_{f}$, then $\partial U$ has no periodic points of $f$.

Proof If $K$ is a boundary component of $U$, then there is $n>0$ such that $f^{n}(K)=K=$ $g^{n}(K)$. Suppose that there is a periodic point $p$ of $f$ in $K$. We assume that $f^{n}(p)=p$ and $f^{n}(U)=U=g^{n}(U)$ by increasing $n$ if necessary. Since $f$ is Moser generic, the first paragraph of the proof of Proposition 4 shows that $p$ must be hyperbolic.

By [3, Proposition 11.1], it follows that the stable and unstable manifolds of $p$ are contained in $K$, so that $K_{p} \doteq K_{p, f}$ is contained in $K$. We will now show that $K_{p}$ has a planar neighborhood. Assume by contradiction that $K_{p}$ has no planar neighborhood, and consider two cases.

Suppose first that $K_{p}, g^{n}\left(K_{p}\right), g^{2 n}\left(K_{p}\right), \ldots$ are pairwise disjoint. Then, for any $m>0$ we can find $m$ disjoint nonplanar manifolds with boundary $N_{1}, \ldots, N_{m}$ in $S$, by taking disjoint neighborhoods of $K_{p}, g^{n}\left(K_{p}\right), \ldots, g^{n(m-1)}\left(K_{p}\right)$. Moreover, we may assume that no component of $\overline{S \backslash N_{i}}$ is a disk, by adding disks to $N_{i}$ if necessary. This implies that each component of $\overline{S \backslash \cup_{i} N_{i}}$ has Euler characteristic at most 0 (for it has at least two boundary components). On the other hand, the Euler characteristic of each $N_{i}$ is strictly negative (because by our assumption they are nonplanar and have nonempty boundary), so we have 


$$
2-2 g=\chi(S)=\chi\left(N_{1}\right)+\cdots+\chi\left(N_{m}\right)+\chi\left(\overline{S \backslash \cup_{i} N_{i}}\right) \leq-m,
$$

where $g$ is the genus of $S$. Since this can be done for all $m$, we get a contradiction.

Now suppose that $g^{k}\left(K_{p}\right) \cap K_{p} \neq \varnothing$ for some $k>0$ multiple of $n$. Then by Proposition 3, we can find $\operatorname{arcs} \gamma_{s}, \gamma_{u} \subset g^{k}\left(W_{f}^{s}(p)\right) \subset K$ such that $\gamma_{s}$ has a nonempty transversal intersection with $W_{f}^{s}(p)$, and $\gamma_{u}$ has a nonempty transversal intersection with $W_{f}^{u}(p)$. By a standard $\lambda$-lemma argument, this implies that, arbitrarily close to $p$, we can find a decreasing sequence of arbitrarily small rectangles $R_{i}$ bounded by arcs in $W_{f}^{s}(p), W_{f}^{u}(p), f^{n_{i} k}\left(\gamma_{u}\right)$ and $f^{-n_{i} k}\left(\gamma_{s}\right)$ (for some $n_{i} \in \mathbb{Z}$ ) accumulating on $p$. Since all of these arcs are contained in $K \subset \partial U$, the same argument used in the proof of Proposition 4 produces a contradiction from the connectedness of $U$. This completes the proof that $K_{p}$ has a planar neighborhood.

But if $K_{p}$ has a planar neighborhood, we know from Proposition 4 that $f$ cannot have periodic points on $K_{p}$, contradicting the definition of $K_{p}$. This completes the proof.

\section{References}

1. Franks, J., Le Calvez, P.: Regions of instability for non-twist maps. Ergodic Theory Dynam. Syst. 23, 111141 (2003)

2. Koropecki, A., Nassiri, M.: Transitivity of generic semigroups of area-preserving surface diffeomorphisms. Math. Z. 266, 707-718 (2010)

3. Mather, J.: Invariant subsets of area-preserving homeomorphisms of surfaces. Adv. Math. Suppl. Stud. 7B, 531-561 (1981)

4. Oliveira, F.: On the generic existence of homoclinic points. Ergodic Theory Dynam. Syst. 7(4), 567595 (1987)

5. Pixton, D.: Planar homoclinic points. J. Differ. Equ. 44(3), 365-382 (1982) 